\title{
Using Intelligent Agents to Manage Business Processes
}

\author{
N. R. Jennings ${ }^{1}$, P. Faratin ${ }^{1}$, M. J. Johnson ${ }^{1}$, P. O’Brien ${ }^{2}$, M. E. Wiegand ${ }^{2}$ \\ ${ }^{1}$ Dept. Electronic Engineering, Queen Mary \& Westfield College, \\ Mile End Road, London E1 4NS, UK. \\ \{N.R.Jennings, P.Faratin, M.Johnson\} @gmw.ac.uk \\ ${ }^{2}$ BT Research Labs, Martlesham Heath, Ipswich, Suffolk IP5 7RE, UK. \\ \{paul, mew\}@info.bt.co.uk
}

\begin{abstract}
This paper describes work undertaken in the ADEPT (Advanced Decision Environment for Process Tasks) project towards developing an agent-based infrastructure for managing business processes. We describe how the key technology of negotiating, service providing, autonomous agents was realised and demonstrate how this was applied to the BT business process of providing a customer quote for network services.
\end{abstract}

\section{INTRODUCTION}

Company managers make informed decisions based on a combination of judgement and information from marketing, sales, research, development, manufacturing and finance departments. Ideally, all relevant information should be brought together before judgement is exercised. However obtaining pertinent, consistent and up-to-date information across a large company is a complex and time consuming process. For this reason, organisations have sought to develop a number of IT systems to assist with various aspects of the management of their business processes. Such systems aim to improve the way that information is gathered, managed, distributed, and presented to people in key business functions and operations. In particular, the IT system should: (i) allow the decision maker to access relevant information wherever it is situated in the organisation (this should be possible despite the fact that information may be stored in many different types of system and in many different information models); (ii) allow the decision maker to request and obtain information management services from other departments within the organisation (and in some cases even from outside the organisation); (iii) proactively identify and deliver timely, relevant information which may not have been explicitly asked for (e.g. because the decision maker is unaware of its existence); (iv) inform the decision maker of changes which have been made elsewhere in the business process which impinge upon the current decision context; and (v) identify the parties who may be interested in the outcome and results of the decision making activity.

Analysis of a number of business processes from various industrial and commercial domains resulted in several common characteristics being identified: (i) Multiple organisations are often involved in the business process. Each organisation attempts to maximise its own profit within the overall activity. (ii) Organisations are physically distributed. This distribution may be across one site, across a country, or even across continents. This situation is even more apparent for virtual organisations [10] which form allegiances for short periods of time and then disband when it is no longer profitable to stay together. (iii) Within organisations, there is a decentralised ownership of the tasks, information and resources involved in the business process. (iv) Different groups within organisations are relatively autonomous - they control how their resources are consumed, by 
whom, at what cost, and in what time frame. They also have their own information systems, with their own idiosyncratic representations, for managing their resources. (v) There is a high degree of natural concurrency - many interrelated tasks are running at any given point of the business process. (vi) There is a requirement to monitor and manage the overall business process. Although the control and resources of the constituent sub-parts are decentralised, there is often a need to place constraints on the entire process (e.g. total time, total budget, etc.). (vii) Business processes are highly dynamic and unpredictable-it is difficult to give a complete a priori specification of all the activities that need to be performed and how they should be ordered. Any detailed time plans which are produced are often disrupted by unavoidable delays or unanticipated events (e.g. people are ill or tasks take longer than expected).

Given these characteristics, it was decided that the most natural way to view the business process is as a collection of autonomous, problem solving agents which interact when they have interdependencies. In this context, an agent can be viewed as an encapsulated problem solving entity which exhibits the following properties [18]:

- Autonomy: agents perform the majority of their problem solving tasks without the direct intervention of humans or other agents, and they have control over their own actions and their own internal state.

- Social ability: agents interact, when they deem appropriate, with other artificial agents and humans in order to complete their problem solving and to help others with their activities. This requires that agents have, as a minimum, a means by which they can communicate their requirements to others and an internal mechanism for deciding what and when social interactions are appropriate (both in terms of generating requests and judging incoming requests).

- Responsiveness: agents perceive their environment and respond in a timely fashion to changes which occur in it.

- Proactiveness: agents do not simply act in response to their environment, they exhibit opportunistic, goal-directed behaviour and take the initiative where appropriate.

The choice of agents as a solution technology was motivated by the following observations: (i) the domain involves an inherent distribution of data, problem solving capabilities, and responsibilities (conforms to the basic model of distributed, encapsulated, problem solving components); (ii) the integrity of the existing organisational structure and the autonomy of its sub-parts needs to be maintained (appeals to the autonomous nature of the agents); (iii) interactions are fairly sophisticated, including negotiation, information sharing, and coordination (requires the complex social skills with which agents are endowed); and (iv) the problem solution cannot be entirely prescribed from start to finish (the problem solvers need to be responsive to changes in the environment and to unpredictability in the business process and proactively take opportunities when they arise). When taken together, this set of requirements leaves agents as the strongest solution candidate(distributed) object systems have the encapsulation but not the sophisticated reasoning required for social interaction or proactiveness, and distributed processing systems deal with the distributed aspect of the domain but not with the autonomous nature of the components.

The remainder of this paper describes the work undertaken to conceptualise business process management as a collection of intelligent agents. Section two describes the key concepts of agents which offer services to one another. Section three details the application of ADEPT agents in BT's customer quote business process. Finally, section four describes the ongoing work and the open issues which still need to be addressed. 


\section{THE BUSINESS PROCESS AS NEGOTIATING AGENTS}

Each agent is able to perform one or more services (figure 1). A service corresponds to some unit of problem solving activity (section 2.2). The simplest service (called a task) represents an atomic unit of problem solving endeavour in the ADEPT system. These atomic units can be combined to form complex services by adding ordering constraints (e.g. two tasks can run in parallel, must run in parallel, or must run in sequence) and conditional control. The nesting of services can be arbitrarily complex and at the topmost level the entire business process can be viewed as a service.

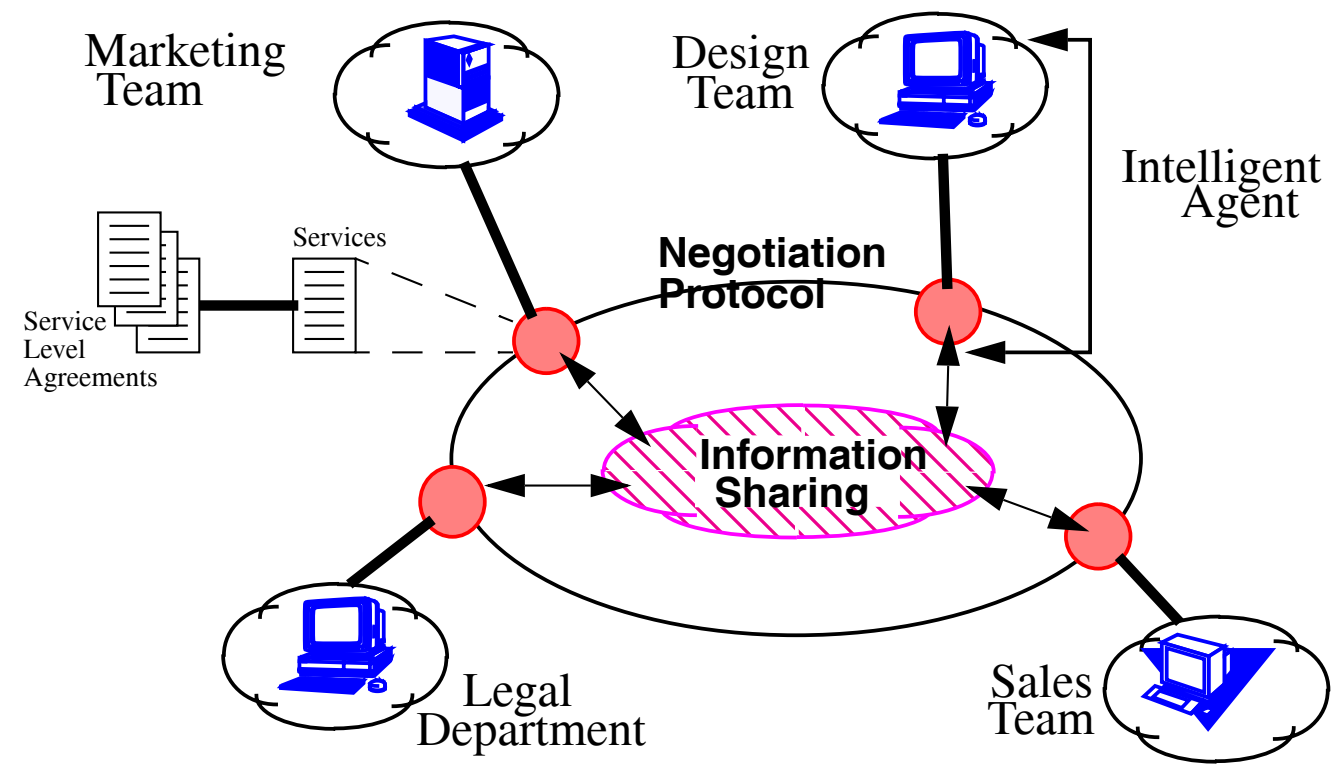

FIGURE 1. An ADEPT Environment

Services are associated with one or more agents which are responsible for managing and executing them. Each service is managed by one agent, although it may involve execution of sub-services by a number of other agents. Since agents are autonomous there are no control dependencies between them; therefore, if an agent requires a service which is managed by another agent it cannot simply instruct it to start the service ${ }^{1}$. Rather, the agents must come to a mutually acceptable agreement about the terms and conditions under which the desired service will be performed (such contracts are called service level agreements (SLAs)—see section 2.3). The mechanism for making SLAs is negotiation - a joint decision making process in which the parties verbalise their (possibly contradictory) demands and then move towards agreement by a process of concession or search for new alternatives [11].

To negotiate with one another, agents need a protocol which specifies the role of the current message interchange-e.g. whether the agent is making a proposal or responding with a counterproposal, or whether it is accepting or rejecting a proposal. Additionally, agents need a means of describing and referring to the domain terms involved in the negotiation-for example, both agents need to be sure they are describing the same service even though they may both have a different (local) name for it and represent it in a different manner. This heterogeneity is inherent in

\footnotetext{
1. This is one of the major features which distinguishes multi-agent systems from more traditional forms of distributed processing [16].
} 
most organisations because each department typically models its own information and resources in its own way. Thus when agents interact, a number of semantic mappings and transformations may need to be performed to create a mutually comprehensible information sharing language (see section 2.4).

\subsection{The ADEPT Agent Architecture}

All ADEPT agents have the same basic architecture (figure 2). This involves an agent head which is responsible for managing the agent's activities and interacting with peers and an agency which represents the agent's domain problem solving resources. The head has a number of functional components responsible for each of it's main activities - communication, service execution, situation assessment, and interaction management (see description below for more details). This internal architecture is broadly based on the GRATE [6, 8] and ARCHON [7] agent models. The domain resources can either be atomic tasks or other agents. The latter case allows a nested (hierarchical) agent system to be constructed in which higher-level agents realise their functionality through lower level agents (the lower level agents have the same structure as the higher level ones and can, therefore, have sub-agents as well as tasks in their agency). For example, the higher level agent may represent a legal department whose work is carried out by a number of lawyers (the lower level agents!). This structure enables flat, hierarchical, and hybrid organisations to be modelled in a single framework ${ }^{2}$. The differences between an agent in an agency and a peer agent relate to the levels of autonomy and helpfulness. In both cases the agents negotiate to reach agreements-however in the former case: (i) the agent cannot reject the proposal outright (although it can counter propose until an acceptable agreement is reached); and (ii) the agent must negotiate in a cooperative (rather than a competitive) manner (since there is some degree of commonality of purpose). In summary, there is a tight coupling between an agent and it's agency and a loose coupling between an agent and it's peers [17].

Communication Module: Routes messages: (i) between an agent and its agency (i.e. between the SEM and the tasks within the agency, between the SEM and agents within the agency during service execution, and between the IMM and the agents within the agency during negotiation); and (ii) between peer agents (i.e. between the SEM and peer agents during service execution and between the IMM and peer agents during negotiation). Communication between the SEM and tasks within the agency relates to task management activities (e.g. activate, suspend, or resume a task), whereas communication between either agents within that agency or peer agents relates to service execution management (e.g. start service, service finished, service results). The IMM's communication both with agency agents and peer agents relates to service negotiation.

Interaction Management Module: Provisions services through negotiation. The SAM invokes the IMM to begin negotiation for services the agent needs. The IMM's decision making capabilities are supported by three types of information: scheduler constraints emanating from the SAM; knowledge an agent has about itself and it's own domain (represented in the SM); and knowledge the agent holds about peer agents (represented in the AM). Based on these sources of knowledge and the negotiation model (section 2.3), the IMM generates initial proposals, evaluates incoming proposals, produces counterproposals, and, finally, accepts or rejects proposals. If a proposal is accepted then the IMM creates a new SLA to represent the agreement.

\footnotetext{
2. This modelling ability is important because commercial environments are founded on organisational models where an enterprise is logically divided into a collection of services. The agent-agency concept draws upon this principle to group services and tasks where it makes pragmatic sense.
} 


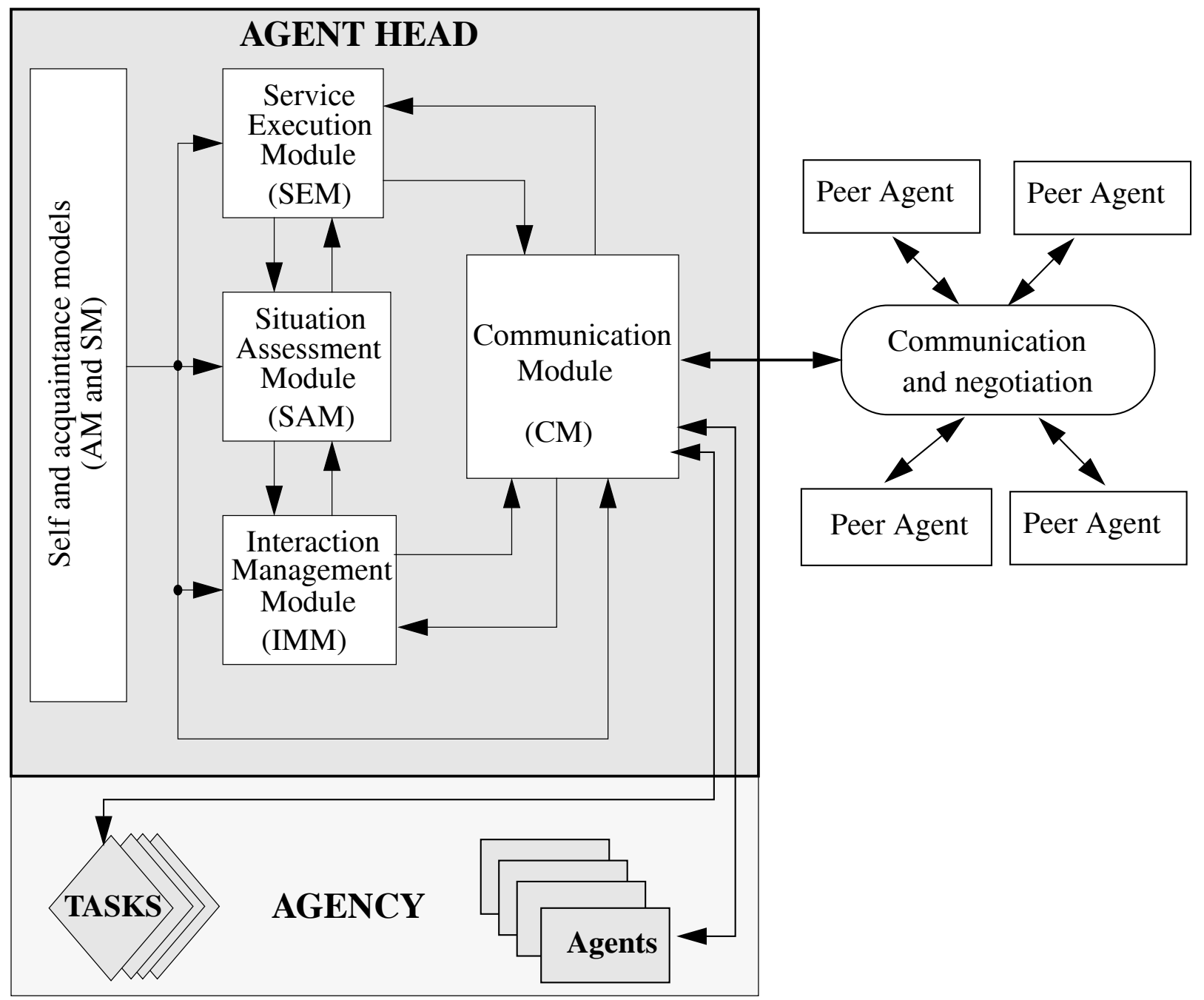

FIGURE 2. The ADEPT Agent Architecture

Situation Assessment Module: Responsible for assessing and monitoring the agent's ability to meet the SLAs it has already agreed and the potential SLAs which it may agree in the future. This involves two main roles: scheduling and exception handling. The former involves maintaining a record of the availability of the agent's resources which can then be used to determine whether SLAs can be met or whether new SLAs can be accepted. The exception handler receives exception reports from the SEM during service execution (e.g. "service may fail", "service has failed", or "no SLA in place") and decides upon the appropriate response. For example, if a service is delayed then the SAM may decide to locally reschedule it, to renegotiate it's SLA, or to terminate it altogether.

Service Execution Module: Responsible for managing services throughout their execution. Involves three main roles: service execution management (start executing services as specified by the agent's SLAs), information management (routing information between tasks, services and other agents during execution), and exception handling (monitor the execution of tasks and services for unexpected events and then react appropriately).

Acquaintance Models: Maintain and provide access to: the SLAs agreed with other agents and a list of peers which can provide services of interest. 
Self Model: Primary storage site for: SLAs to which the agent is committed; descriptions of the services the agent can provide; run time application/service specific information (e.g. the services which are currently active and the current number of invocations of each active service), and generic domain information (e.g. the upper limit the agent will pay for a service and the maximum permissible number of concurrent invocations of each service).

\subsection{The Service Lifecycle}

There are three distinct phases to the service lifecycle (figure 3). Firstly, the agent programmer has to describe the service and how it is realised. This is carried out using ADEPT's service description language (SDL). As an illustration, figure 4 shows a service description from the customer quote business process (section 3). A service is described by a name, its inputs, its outputs, and its body. The name uniquely identifies the service within the particular agent in which it is situated. The input field specifies what information is needed by the service, who is to provide it, and whether it is mandatory (must be provided before the service can start) or optional (if available it will be used, but if it is unavailable the service can still proceed). In the example shown, the service must have both of its inputs available: cr_profile of type Bt_CrProfile ${ }^{3}$ from the client agent and cust_details of type Bt_CustomerDetails from the server agent. The output field specifies the information produced by the service (in this case it is network_design which is of type Bt_NetworkDesign and detailed_reqs which is of type Bt_CustomerReqs). The body specifies the way the service is realised (i.e. which services and tasks need to be performed, their partial order, and the information shared between them) and the conditions which prevail if it is successful (the construct specifying this is the completion expression $)^{4}$. In the example shown, the mainblock of the service consists of three subservices (task_analyse_reqs, subblock and task_design_network) which need to be executed in sequence. Associated with mainblock is a completion expression, (and task_analyse_reqs subblock task_design_network), which specifies that each of the sub-services must successfully complete if the whole service is to succeed.

The first sub-service to be executed is task_analyse_reqs which takes cr_profile as its input and produces as its output detailed_reqs and SurveyReqd. When this sub-service finishes, the completion expression within which it was invoked is evaluated. If task_analyse_reqs fails (i.e. the requirements cannot be analysed) then the whole network design service is terminated since the completion expression (a conjunction) will necessarily fail. In this case, the remaining sub-services are not executed. If task_analyse_reqs succeeds, then the overall completion expression is still evaluated. However, in this case it's value is unknown since although task_analyse_reqs is true the values of the other two services in the conjunction are unknown at this point (the conjunction of the truth values true and unknown is unknown).

\footnotetext{
3. The various types of information are defined in the agent's information model. Creating an agent involves determining the information model to be used as well as specifying the service details. However this aspect of agent creation is not elaborated upon here.

4. A procedural language is not used because such languages typically require a rigorously specified flow of control. Since the body is executed by an autonomous agent in an unpredictable environment, it is felt that such control decisions are best left to the agent to determine at run-time (rather than being dictated by the designer at compile time). Thus, in the ADEPT SDL, the body specifies a partial flow of control with some restrictions on the order and the degree of concurrency of the execution and the completion expression supplies the agent with the completion logic of the block (in terms of success, fail, and unknown). It is then up to the agent to complete the service by the most appropriate means given its current circumstances.
} 


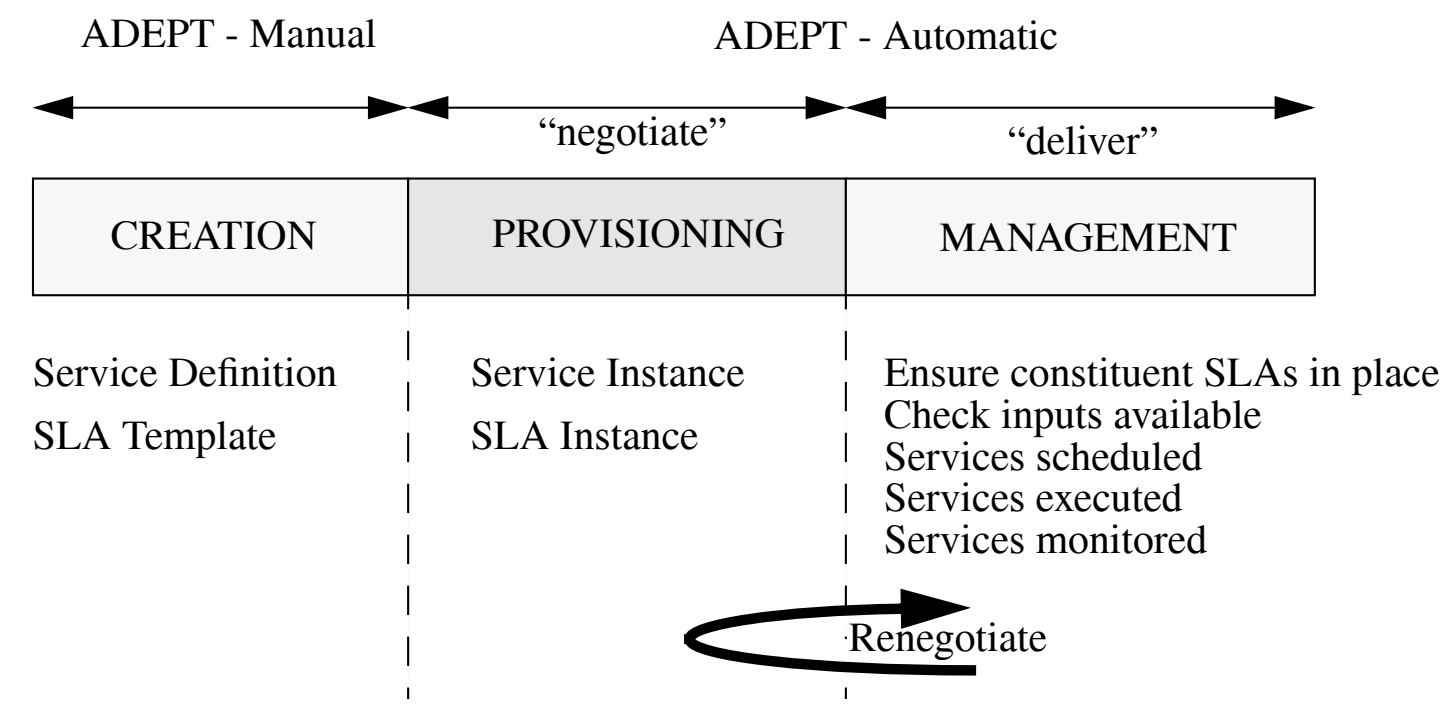

FIGURE 3. The Service Lifecycle

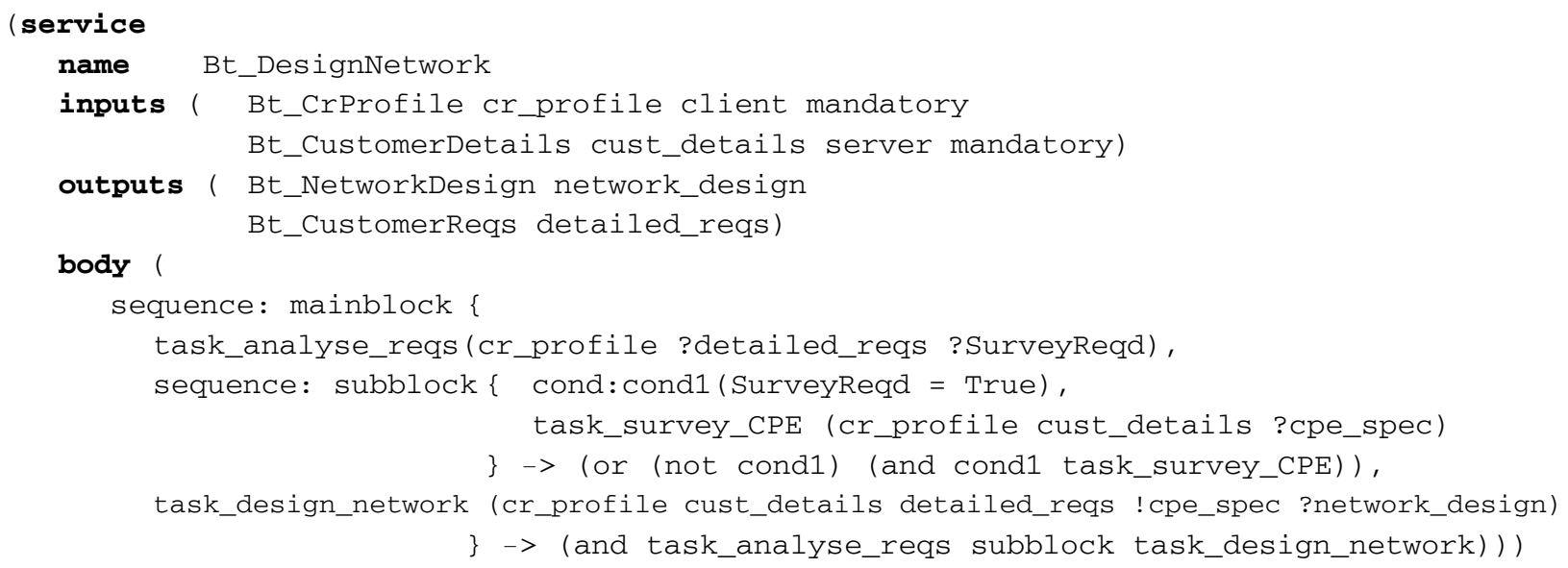

FIGURE 4. Sample SDL Description

Assuming the requirements are successfully analysed, the next sub-service is executed. Subblock is a composite construct involving two sequential actions. The first component is a conditional statement which must evaluate to true before the second component (task_survey_CPE) is performed. The conditional tests whether a survey is required. If a survey is not needed (i.e. the conditional is false) then the completion expression in the subblock is satisfied since (not cond1) is true (the completion expression is a disjunction). Control then switches to task_design_network. Alternatively, if a survey is needed then cond1 is true and hence the completion expression's first disjunct is false. Since the second disjunct remains unknown, subblock does not fail at this point. Subblock's second sub-service, task_survey_CPE, is then executed. This service takes two inputs_cr_profile and cust_details-and produces cpe_spec. If task_survey_CPE is successful, the second disjunct in subblock's completion expression is satisfied which means that subblock succeeds.

If subblock succeeds, task_design_network is executed. This takes as its input cr_profile, cust_details, detailed_reqs and cpe_spec (optional input) and produces as it's output network_design. If this sub-service completes then mainblock completes since the conjunction of the three sub-services is now true. 
Once a service has been created and placed within an agent it becomes accessible to the other agents in the system. To activate a service, the client and the server agents negotiate until they come to a mutually acceptable SLA—no service can be executed without a concomitant SLA being in place. An important facet of this negotiation is the manner in which the service is provisioned. ADEPT supports three different provisioning modes depending on the client agent's intended pattern of usage and the server agent's scheduling capabilities: (i) One-Off: the service is provisioned each and every time it is needed and the agreement covers precisely one invocation; (ii) Regular: the service is required a number of times, but it is known in advance when it is needed; and (iii) On-Demand: the service can be invoked by the client on an as needed basis within a given time frame (subject to some maximum volume measurement specified in the SLA).

If the provisioning phase is successful, a specific instance of the service is created for execution within the context of an associated SLA instance. At some point the agent needs to execute the service, this requires it to ensure: that appropriate SLAs are in place for constituent sub-services, that the required input information is available, that the service is scheduled so that any constraints specified in the SLA are met, and, ultimately, that the appropriate services and tasks are executed (either within the local agency or by the chosen peer agent). Since the agent is situated in a dynamic and unpredictable environment, it must keep track of it's context-thus new services may be agreed which require the agent to reschedule its resources or currently scheduled services may fail and require the agent to replan its execution strategy. In the extreme case, the agent may even need to return to the provisioning phase to renegotiate a SLA which cannot be satisfied in the current situation.

\subsection{The Negotiation Model}

There are three components of the ADEPT negotiation model-the protocol, the service level agreements, and the reasoning model. The protocol itself is relatively standard and is based on speech-act performatives $[2,15]$. It covers the process of finding out the services an agent can perform (agent sends out a CAN-DO primitive and respondents return a I-CAN primitive), the provisioning phase of coming to an agreement (PROPOSE, COUNTER-PROPOSE, ACCEPT, and REJECT), and the management phase of actually invoking the agreement (ACTIVATE-SERVICE, SUSPEND-SERVICE, RESUME-SERVICE, SERVICE-FAILED, SERVICE-COMPLETED)—see [1] for more details of this work. The novel aspects of negotiation in the ADEPT system relate to the types of agreements which agents can make and the models they use to guide their negotiation behaviour. The requirements of the business process domain mean that agreements need to be more encompassing and the reasoning more elaborate than those found in most extant multi-agent systems.

The nature and scope of the SLAs are derived almost exactly from the types of legal contract which are often used to regulate current business transactions (figure 5). Service_name is the service to which the agreement refers and sla_id is the SLA's unique identifier (covering the case where there are multiple agreements for the same service). Server_agent and client_agent represent the agents who are party to the agreement. Delivery_type identifies the way in which the service is to be provisioned (section 2.2). The SLA's scheduling information is used by the SAM and the SEM for service execution and management-duration represents the maximum time the server can take to finish the service, and start_time and end_time represent the time during which the agreement is valid. In this case, the agreement specifies that agent CSD can invoke agent DD to cost and design a customer network whenever it is required between 09:00 and 18:00 and each service execution should take no more than 320 
minutes. The agreement also contains meta-service information such as the volume of invocations permissible between the start and end times, the price paid per invocation, and the penalty the server incurs for every violation. Client_info specifies the information the client must provide to the server at service invocation (in this case CSD must provide the customer profile) and reporting_policy specifies the information the server returns upon completion.

\begin{tabular}{|c|c|}
\hline Slot Name & Instantiated Values \\
\hline SERVICE_NAME: & cost_\&_design_customer_network \\
\hline SLA_ID: & a1001 \\
\hline SERVER_AGENT: & $\mathrm{DD}$ \\
\hline CLIENT_AGENT: & CSD \\
\hline SLA_DELIVERY_TYPE: & on-demand \\
\hline DURATION: (minutes) & 320 \\
\hline START_TIME: & $9: 00$ \\
\hline END_TIME: & $18: 00$ \\
\hline VOLUME : & 35 \\
\hline PRICE: (per costing): & 35 \\
\hline PENALTY : & 30 \\
\hline CLIENT_INFO: & cr_profile \\
\hline REPORTING_POLICY: & customer_quote \\
\hline
\end{tabular}

FIGURE 5. Exemplar Service Level Agreement

The reasoning model also represents a novel contribution of this work. Existing work on negotiation can be divided into two distinct camps. The theoretical work (e.g. [12, 13, 14]) provides important insights into how agents should negotiate to produce optimal solutions. However, a number of unrealistic assumptions are common in these negotiation models; typical assumptions include the availability of complete action descriptions, a utility function that can order all alternatives in all contexts, and that agents exhibit perfect rationality when selecting actions. In contrast, the practical work typically adopts a very superficial approach to negotiation. In the much vaunted contract net protocol [16], for instance, a manager sends out a request to a number of potential contractors to provide a given service to a given degree of quality. The potential contractors return a bid if they are capable of fulfilling all the requirements. The manager then selects the best bid. This model fails to capture many intuitive and important aspects of the negotiation process. For example, bidders cannot counter-propose better options, they cannot modify any of the service agreement parameters, and the emphasis in devising a complete specification is placed solely with the task manager.

The approach within ADEPT is to develop a deep and explicit model of the process of negotiation (this terminology is analogous to its use in the context of reasoning models for second generation expert systems $[3,4,9])$. Such a model is needed to capture the richness of the interactions which take place when setting up agreements in this domain. The model covers the whole process of generating initial offers, evaluating offers, and counter proposing if offers are unacceptable. 
The model has two component knowledge bases: a declarative one and a procedural one. The former, represented as a causal network, explicitly models what is being negotiated for and why the negotiation is taking place (i.e. it sets the negotiation context). For example, negotiation over the price of a service is a meta-service conflict that can be caused by an agent believing it is being over charged. Similarly, an agent may need to negotiate over a service's start time if the client's proposal conflicts with it's existing commitments. The procedural knowledge base, represented as a set of strategies and mechanisms for selecting between them, specifies which actions should be taken given the declarative knowledge. For example, given that the agent needs to negotiate over price, the knowledge base may indicate that Boulware 5 is a good strategy to adopt if the agent has a long time to reach an agreement or if there are many potential suppliers of the service. In such cases, the agent generates a price offer and continues to counter-propose that initial offer throughout the negotiation. Alternatively, if the agent wants to reach an agreement for a scarce service or if it is negotiating with an agent in it's agency, then it may adopt the more cooperative tit-for-tat strategy-making concessions when the agent concedes and standing firm when the other agent is uncompromising.

\subsection{Information Sharing}

Agent negotiation requires a reliable means of communication. Such communication can be viewed on two levels: (i) actually transporting the messages; and (ii) conveying the desired meaning of the message. The former is handled transparently by the agent's underlying infrastructure. The latter is more problematic and requires conceptual design. Because of the characteristics of the business process domain (section 1), it is impractical to insist that all agents conform to a common model of information. For example, a surveyor may find it necessary to represent location information in terms of grid references on a particular map, but within the customer liaison department a location may be described in terms of an address. If autonomous agents representing these two departments are to communicate such information, they must be aware of the differences in their models of information.

The information contained within a message must be understood by both sender and recipient. For this reason, agents within an ADEPT environment must transform information represented in their local form into a common communication language. This language is still under development, but will consist of a number of semantically grounded speech acts $[2,15]$ which will specify the intention of the message, and a KIF-like [5] syntax (i.e. an extended first-order predicate calculus) which will express the content of the message. Furthermore, the recipient must be able to understand the meaning of the symbols contained in the message. Suppose that the agent sending the message uses model $\mathrm{A}$ and the recipient uses model $\mathrm{B}$, then the information contained in the message must be translated between these models for the agents to understand each other. For example, an address must be translated into an appropriate grid reference for an agent representing the customer liaison department to communicate location information to an agent representing a surveyor.

\section{BT'S CUSTOMER QUOTE BUSINESS PROCESS}

This scenario is based on BT's business process of providing a quotation for designing a network to provide particular services to a customer (figure 6$)^{6}$. The process receives a customer service

\footnotetext{
5. Boulwarism is the strategy in which the negotiator makes a reasonable initial offer and then sticks firm throughout the negotiation [13].
} 
request as its input and generates as its output a quote specifying how much it would cost to build a network to realise that service. It involves up to six parties: the sales department, the customer service division, the legal department, the design division, the surveyor department, and the provider of an out-sourced service for vetting customers.

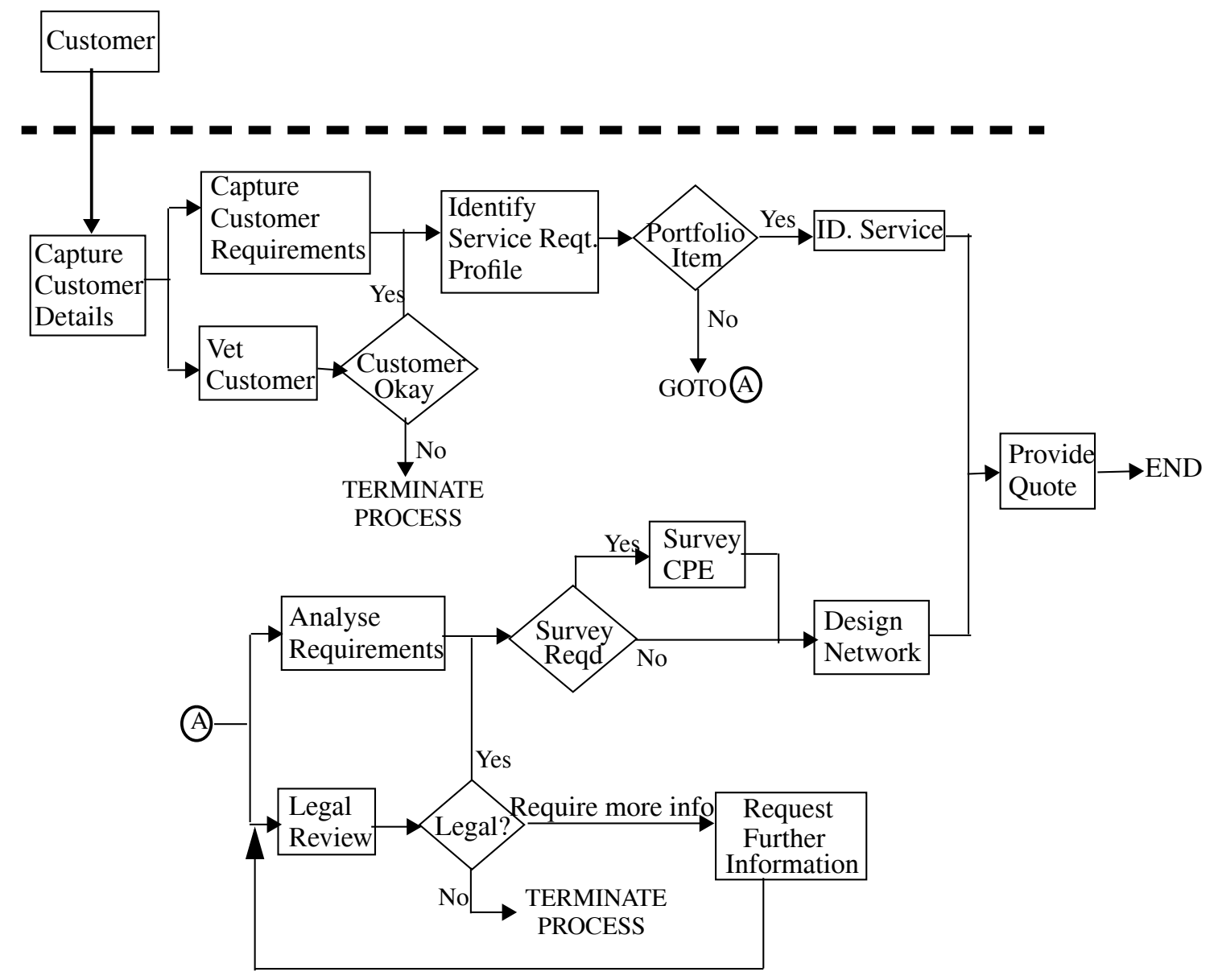

FIGURE 6. The Provide Customer Quote Service

The process is initiated by a customer contacting the customer service division. The customer's details are captured and whilst the customer is being vetted (in terms of its credit worthiness, false ID, etc.) their requirements are ellicted. If the customer fails the vetting procedure, then the quote process terminates. Assuming the customer is satisfactory, it's requirements are recorded and mapped against the service portfolio. If the requirements can be met by a standard off-the-shelf portfolio item then an immediate quote can be offered based on previous examples. In the case of bespoke services, however, the process is more complex and involves a bid manager. The bid manager further analyses the customer's requirements and whilst this is occurring the legal department checks the legality of the proposed service (e.g. it is illegal to send unauthorised encrypted messages across France). If the desired service is illegal, then the entire quote process terminates and the customer is informed. If there is any uncertainty about the service's legality,

\footnotetext{
6. The scenario has been simplified for the purposes of explanation and demonstration. The real business process for this service contains 38 tasks and 9 choice points. Despite this simplification, the key aspects of the process are still present. Each activity requires resourcing and has a start/end point whereby progress can be measured. Choice points indicate which sequences of activities require provisioning. There are a number of concurrent activities which require coordination.
} 
then the business process is suspended while further information is obtained from the customer. If the requested service is definitely legal then the design phase can start. To prepare a network design it is usually necessary to have a detailed plan of the existing equipment at the customer's premises (CPE) - the exception to this is when the desired service is sufficiently simple that a survey is not warranted. Sometimes such plans might not exist and sometimes they may be out of date. In either case, the bid manager determines whether the customer site(s) should be surveyed. On completion of the network design and costing, the customer is informed of the service quote and the business process terminates.

From the business process description, the following agent system was designed (figure 7). The agents (denoted by the circles) were chosen to represent distinct departments or enterprises involved in the customer quote business process. The VC agents represent the concerns of external enterprises as this activity is outsourced. Agent SD is within DD's agency because the design division has overall management responsibility for the surveyor department (i.e. all requests for site surveys must be channelled through the design department).

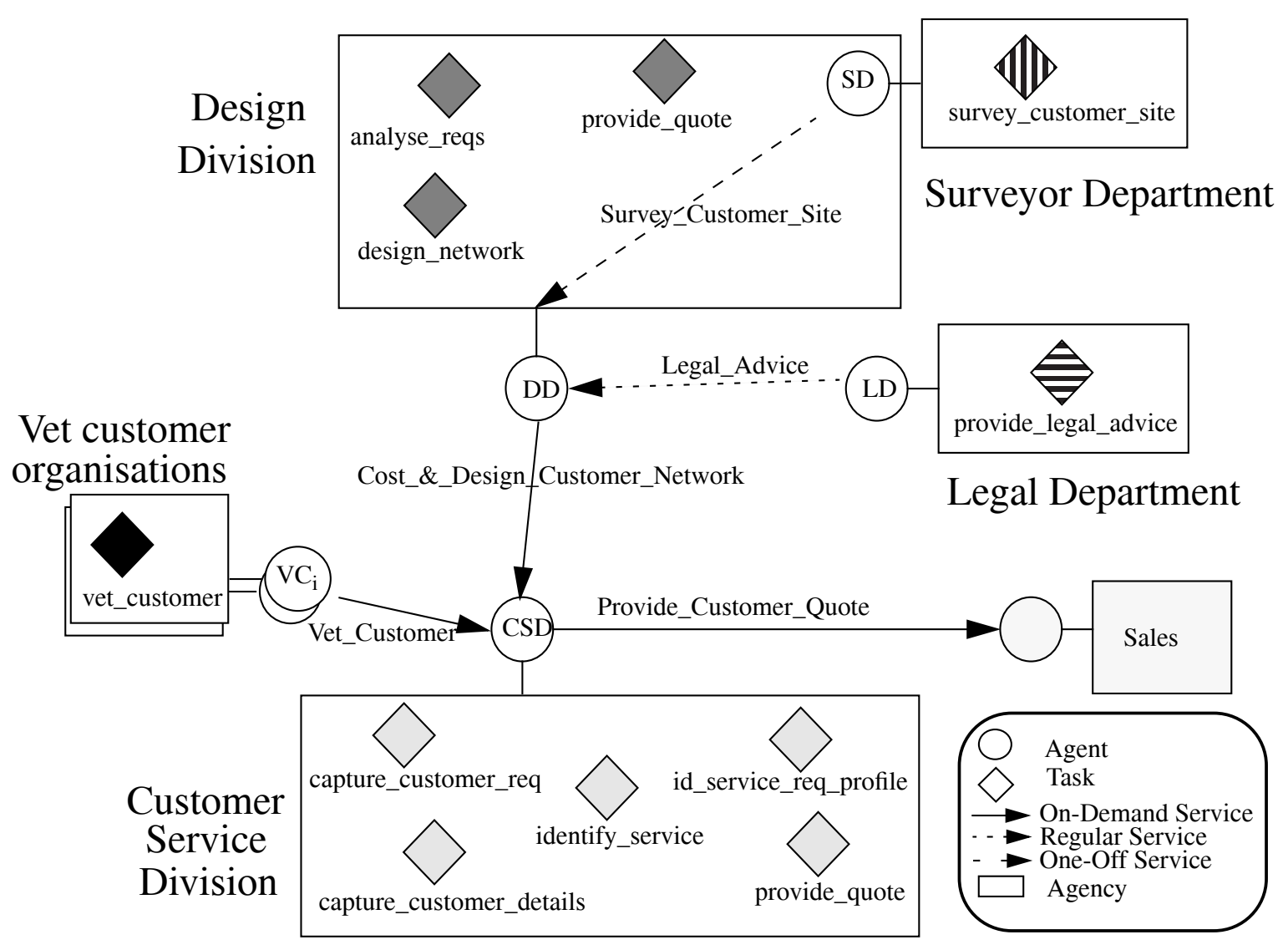

FIGURE 7. Agent System for the Provide Customer Quote Business Process

The process is triggered when the sales agent sends a request to the CSD agent to provide a customer quote ${ }^{7}$. The CSD agent identifies the SLA associated with the request-in this case it relates to the Provide_Customer_Quote service. The SDL body of Provide_Customer_Quote is parsed to create a tree of possible routes that the SEM can take. A depth first path is selected and the tasks and services in that path are scheduled and

7. The scenario assumes an SLA between the CSD and the sales department has already been negotiated. 
resourced (by the SAM). The SEM begins executing the constituent sub-services and tasks. One of the first sub-services it encounters is to vet the customer (this occurs in parallel with the capture_customer_req task and after capture_customer_details). When the SEM comes to execute this service it realises (by checking it's SM) there is no associated SLA and so it reports an exception to the $\mathrm{SAM}^{8}$. The SAM determines that the service cannot be realised locally (by referring to its SM) and so it must be bought in from an external agent. It also decides that the service should be provisioned in an on-demand manner because it is an activity which is needed on each invocation of the business process. As such, it is preferable to negotiate for a longer term SLA covering multiple invocations rather than negotiating for one each time the business process is invoked. In addition to identifying the service name and the desired provisioning mode, the SAM indicates any scheduler information which influences the service's provisioning (e.g. the service's earliest start and latest end times).

Vet customer service provisioning begins with the CSD's IMM sending CAN-DOs to all the agents it can identify (using its $\mathrm{AM}$ ) as being potentially able to provide this service (in this scenario there are three such agents $-\mathrm{VC}_{1}, \mathrm{VC}_{2}$ and $\mathrm{VC}_{3}$ ). Negotiation proper begins when the CSD agent concurrently sends out initial proposals (in the form of instantiated SLAs) to all the vet customer agents which responded with I-CAN. This initial proposal may be acceptable to one of the $\mathrm{VC}$ agents in which case an agreement is made and the negotiation is terminated. However, in most cases the VC agents find some part of the proposal unsatisfactory and so return a revised counterproposal to CSD. The CSD and VC agents then engage in several concurrent rounds of exchanging SLA messages until either the CSD comes to an agreement with one of the VC agents or all the VC agents reject all the offers and break off negotiation. If the CSD agent receives more than one acceptable offer, it selects the one closest to it's specified optimum ${ }^{9}$. The chosen agent is informed of it's success and an SLA for the Vet_Customer service comes into force. The CSD's IMM then informs its SAM which, in turn, reinvokes the SEM's execution of Provide_Customer_Quote with the freshly agreed Vet_Customer SLA stored in its SM. Since the agreement is for on-demand provisioning - the CSD can ask the chosen VC to vet customers as and when new customers are presented to it from the sales department. The CSD's SEM sends a SERVICE-ACTIVATE request to the SEM of the selected VC within the time frame specified in the SLA. When the customer has been vetted, the client VC agent informs the CSD's SEM of the result (as specified by the SLA's reporting policy). If the customer fails the vetting procedure then Provide_Customer_Quote fails and the sales department is informed. If the customer is successfully vetted, the CSD's SEM starts executing the next sub-service.

The next sub-service checks whether the customer's request is for a portfolio item-achieved by executing the id_service_req_profile task. If it is a portfolio item then the service is identified (identify_service) and a quote is looked up (provide_quote) and returned to the sales department (as specified in the SLA between the CSD and the sales department). Execution of Provide_Customer_Quote then terminates.

\footnotetext{
8. In future versions of the system, agents will pre-parse the SDL body to see which SLAs need to be set up before it comes to their actual execution. This will allow the agents to proactively negotiate SLAs. In this case, agents need to strike a balance between expending resources provisioning services which may not be used and only provisioning services when they are actually needed (which may delay their execution).

9. If the negotiation fails to find any agents willing to vet customers, the CSD's SAM is informed and that particular invocation of Provide_Customer_Quote fails. In this case, service failure is reported back to the sales department. For the future, we are investigating techniques for dynamically revising the business process in such situations.
} 
If the desired service is bespoke-id_service_req_profile fails-then the next sub-service to be executed is Cost_\&_Design_Customer_Network. Again the SEM informs the SAM that there is no associated SLA in place. The SAM decides the service must be bought in (after examining its SM) and that it should be provisioned in an on-demand manner (because it is required every time a customer requests a bespoke service. A one-off SLA would be justified if a significant proportion of the customer service requests were for portfolio items). It then asks the IMM to obtain an appropriate agreement. The IMM notes from its AM that the only agent offering this service is DD and so it starts negotiating with it. Assuming the two agents reach an agreement, the CSD's IMM informs it's SAM which informs it's SEM that an appropriate SLA is now in place (see figure 5). When the CSD's SEM indicates that the Cost_\&_Design_Customer_Network service should be invoked, the DD agent starts executing it under the newly agreed SLA. This service involves executing the Bt_DesignNetwork sub-service (figure 4) to produce the network design, ensuring the necessary legal checks are performed, and executing the provide_quote task to cost the design. When the customer's requirements have been analysed in more detail (i.e. detailed_reqs are available from Bt_DesignNetwork), the legality of the customer's request is checked ${ }^{10}$. The DD agent realises (by checking its AM) this service can only be provided by the LD agent and so it starts to negotiate with it. The service is provisioned in a one-off manner because it too expensive to have waiting idle when there are no designs to check. When the agreed legal service is invoked, the requirements are checked and the appropriate course of action is taken depending on the outcome of this review.

As part of Bt_DesignNetwork, a survey of the customer's premises may be needed. If this is the case, the DD's SEM informs it's SAM that no SLA is in place for task_survey_CPE. The SAM notes (by examining its AM) that an agent (SD) within it's agency can provide the service. It decides the service should be provisioned in a one-off manner (because the service is only occasionally required) and so the DD's IMM negotiates with SD. Assuming they reach an agreement, the DD agent invokes the agreement and requests SD to obtain a survey for the customer's premises. When the survey is complete or after the service is declared legal if no survey is required, the network design is carried out and then costed. The service's cost is returned to the CSD agent as specified in the Cost_\&_Design_Customer_Network SLA (figure 5). The Provide_Customer_Quote service then completes and the quote is returned to the sales department as specified in the SLA with the sales department.

For subsequent service quote requests, several of the basic agreements for managing the business process are already in situ. The CSD agent has an on-demand SLA for vetting customers and it may also have an agreement for costing and designing the customer's network. This means there is less of a negotiation overhead on subsequent process invocations. The services which may generate further negotiations in subsequent quote processes are those which are only occasionally invoked-legal services and survey customer site.

\section{CONCLUSIONS \& FUTURE WORK}

This paper has described the key components of the ADEPT system and how they were applied to BT's business process of providing a customer quote for network services. This work can be

10. Checking is managed in Cost_\&_Design_Customer_Network by having a completion expression which either stops or suspends the design activity if the customer's request is not legal or allows it to continue if the request is legal. 
viewed on three different levels, each of which represents increasing support for the realisation of business process management software systems:

- ADEPT as a design technology. ADEPT proposes a method of approach for structuring the design and development of business process management systems. It identifies the key concepts in this view as autonomous agents, negotiation, service provision, service level agreements, resource management, and information sharing. This view can be readily applied to other business process applications without being tied to the details of how they were realised in ADEPT.

- ADEPT as an implementation technology. As well as identifying a methodology, the ADEPT system provides algorithms, interfaces, language definitions, and structures for realising the key concepts. These definitions can be re-implemented in other programming environments to develop ADEPT-like agent systems for business process management.

- ADEPT as a solution technology. The ADEPT programming environment can be re-used in other business management applications. In this case, the ADEPT design methodology is used to structure the application and the ADEPT software is used to implement it.

Implementing the scenario has also identified a number of system and agent issues which require further investigation - these include: the need for richer and more flexible negotiation models, more scalable techniques for sharing information between heterogeneous agents, more elaborate resource management within agents, and more flexible service scheduling algorithms.

\section{ACKNOWLEDGEMENTS}

ADEPT is a collaborative project under the DTI/EPSRC Intelligent Systems Integration Programme (ISIP). The project partners are BT Laboratories, ICI Engineering, Loughborough University of Technology, and Queen Mary and Westfield College. The work described in this paper represents a partial view of the activities of the whole project to which all consortium members have contributed. The authors would like to thank Tim Norman for his comments on earlier drafts of this paper and for providing the material on information sharing.

\section{REFERENCES}

[1] J. L. Alty, D. Griffiths, N. R. Jennings, E. H. Mamdani, A. Struthers, and M. E. Wiegand (1994) "ADEPT - Advanced Decision Environment for Process Tasks: Overview \& Architecture" Proc. BCS Expert Systems 94 Conference (Applications Track), Cambridge, UK, 359-371.

[2] J. L. Austin (1962) "How to do Things with Words" Harvard University Press.

[3] B. Chandrasekaran (1983) "Generic Tasks in Knowledge Based Reasoning: High Level Building Blocks for Expert System Design” IEEE Expert 1 (3) 23-30.

[4] W. J. Clancy (1985) “Heuristic Classification” Artificial Intelligence 27 (3) 289-250.

[5] M. R. Genesereth and R. E. Fikes (eds.) (1992) "Knowledge interchange format, version 3 reference manual" Computer Science Department, Stanford University, Technical Report Logic-911. (http://www-ksl.stanford.edu/knowledge-sharing/papers/index.html). 
[6] N. R. Jennings (1995) "Controlling Cooperative Problem Solving in Industrial Multi-Agent Systems using Joint Intentions” Artificial Intelligence 75 (2) 195-240.

[7] N. R. Jennings, J. Corera, I. Laresgoiti, E. H. Mamdani, F. Perriolat, P. Skarek and L. Z. Varga (1996) "Using ARCHON to develop real-word DAI applications for electricity transportation management and particle accelerator control" IEEE Expert.

[8] N. R. Jennings, E. H. Mamdani, I. Laresgoiti, J. Perez and J. Corera (1992) "GRATE: A General Framework for Cooperative Problem Solving" IEE-BCS Journal of Intelligent Systems Engineering, 1 (2) 102-114.

[9] J. McDermott (1988) "A Taxonomy of Problem Solving Methods" in Automating Knowledge Acquisition for Expert Systems (ed S. Marcus) Kluwer 225-256.

[10] A. Mowshowitz (1986) "Social Dimensions of Office Automation" Advances in Computers, $25,335-404$.

[11] H. J. Mueller, (1996) "Negotiation Principles" in Foundations of Distributed Artificial Intelligence (eds. G. M. P. O’Hare and N. R. Jennings) Wiley Interscience.

[12] J. F. Nash, (1950) “The Bargaining Problem” Econometrica 28: 155-162.

[13] H. Raiffa (1982) “The Art and Science of Negotiation” Harvard University Press.

[14] J. S. Rosenschein and G. Zlotkin (1994) "Rules of Encounter - Designing Conventions for Automated Negotiation Among Computers", MIT Press.

[15] J. R. Searle (1969) "Speech Acts: An Essay in the Philosophy of Language" Cambridge University Press.

[16] R. G. Smith and R. Davis, (1981) "Frameworks for cooperation in distributed problem solving” IEEE Trans on Systems, Man and Cybernetics 11 (1) 61-70.

[17] N. S. Sridharan, (1987) “1986 Workshop on Distributed AI” AI Magazine, Fall, 75-85.

[18] M. J. Wooldridge and N. R. Jennings, (1995) "Intelligent Agents: Theory and Practice" The Knowledge Engineering Review 10 (2) 115-152. 